Global Conferences Series:

Social Sciences, Education and Humanities (GCSSSEH), Volume 3, 2019

The $1^{\text {st }}$ International Conference on Education, Social Sciences and Humanities

DOI: https://doi.org/10.326/hum0192

\title{
Developing Instruments for Need Assessment in Crisis Counseling Programs
}

\author{
Najlatun Naqiyah ${ }^{1}$, Evi Winingsih ${ }^{2}$, Siti Ainiyah ${ }^{3(*)}$ \\ 1,2 Universitas Negeri Surabaya \\ ${ }^{3}$ Senior High School 2 Surabaya \\ (*) $\bowtie$ najlatunnaqiyah@unesa.ac.id
}

\begin{abstract}
The purpose of this research is to develop a measuring instrument used to analyze the needs of students. The very instrument is designed for crisis counseling and guidance programs. It is utilized to map out anxiety on counselees those are in a crisis situation. There are 7 indicators developed in it, including aspects of psychological development, violence, broken family, twisted moral and religious values, bullying in schools, suffering for HIV/AIDS, and busy working parents. Tests for validity of the data are in two stages, that are a test for construct validity and a test for empirical one. Test for construct validity is undertaken through setting appropriate indicators which are in accordance to a theoretical point of view. Whereas a test for empirical validity is obtained through testing on data collecting out of 75 high school students in Surabaya. The test for validity and its reliability uses the Cronbach's Alpha method. The test produces 20 items that are valid. Need assessment in crisis counseling can be used by counselors to map out the students at the beginning sessions of individual and group counseling. The results of this need assessment help the counselor to set goals and strategies along with the counselees.
\end{abstract}

Keywords: need assessment, crisis, counseling

\section{Introduction}

Adolescence is a period for searching an identity. Adolescence is not a homogeneous developmental phase but rather involves a collection of developmental changes that are unique to each teenager (Foa, Chrestman, \& Gilboa-Schechtman, 2008). They need guidance in order to actualize themselves and to obtain a positive explanation. Teenagers tend to sink into crisis of moral degradation, uncontrolled lifestyles, peer pressure, parents' busy life, and social demands that all of which lead them to have crises (Kopetz et al., 2019). Therefore, prevailing problems on adolescence need to be identified as early as possible.

Developing a crisis need assessment in this article could detect anxiety disorders experienced by teenagers during their crisis. By knowing the problem of the crisis that befell on the counselee, the counselor is able to help the individuals to overcome the crisis, therefore they develop a positive identity. Moreover, individual's needs are various. As their needs are not met, they become depressed. Therefore, identification for their need should be known. Developing the crisis need

Copyright (C) 2019, the Authors. Published by Redwhite Press. 
assessment will encourage self-understanding. Thus they seek to overcome the problems through improving the situation and avoiding risks (Novita Luthfiana \& Psi, 2017).

Individuals who have crises will feel anxious, upset, sad, suffer and hopeless. This crisis brings them into an unwanted behavior. Self-destructive actions such as blaming others, spreading hatred, despair, running into promiscuity, being lazy, theft and other criminal acts will bring them into the gravely suffering. The habit of playing gadgets, hanging out in coffee shops, lazy to study, spending time in vain, and live without schedule become a symptom of bad developmental adolescent. They are confused in the mid of rapid change, and only become spectators of change.

The involvement of parents is needed in children's education. However, the available reality shows that many parents busily work to meet the family needs (Bronfenbrenner, 1986). Their busy and their long distance to their children creates pressure. Their stress increases. They require the quality of meeting between children and parents (Maté \& Neufeld, 2019).

Conversely individuals who are able to achieve developmental identity come to strong independent, and dare to avoid risk. Strengthening for this individual is a compulsory so that they are able to adapt to the fast changing world, that is the industrial era 4.0. In the era human powers are replaced by robotic power and artificial intelligence. Many jobs are erased. As a result, unemployment increases. Therefore, individual values need adjustment so as to respond to the industrial era 4.0. Cognitive, emotional, and environmental relationships become reciprocal relationships in response to the crisis. Every individual should continue to adjust to the change. Innovation and creativity are keys to brim them able to adapt to the industrial age 4.0 as the situation is more difficult and much of competition.

The purpose of this study was to develop a need assessment for the crisis. What bring individuals into crisis in the era 4.0? How to map out the individual crisis problems? What should be developed to help individuals as they face a crisis situation? What are the implications for counselors in providing crisis counseling services at school?

\section{Method}

This research method uses a quantitative approach. This research was conducted in high school which has a culture of discipline and high achievement. Sampling in this study was carried out by convenience sampling technique (also known as Sampling Haphazard or Accidental Sampling). The Convenience sampling is a type of impossibility or nonrandom sampling in which the target population meets certain practical criteria, such as easy accessibility, geographical proximity, availability at a certain time, or willingness to participate for the purpose of this study (Ethics, Moses, \& Alkassim, 2016). The population of class XI students by which this research conducted is 37, while the sample is 75 students.

Tests for the validity are carried out through construct validity and empirical validity. Construct validity is a validity which examine how far the test items are able to measure in accordance with a specific concept or conceptual definition that has been set (Matondang, 2009). To determine construct validity, the theoretical review process of the concept is carried out from the variables measured that starting from the formulation of constructs, determining dimensions and indicators, to the elaboration and writing of items of the instrument. Formulation, the construct should be based on a synthesis of theories about the concept of variables that are to be measured through a logical and careful process of analysis and comparison (Matondang, 2009). A test is said to be valid if it conforms criteria (Malawi \& Maruti, 2009). Reliability test is done by using alpha croanbach to measure the stability of the instrument.

\section{Results and Discussion}

Measurement results for the developing need crisis assessment for guidance and counselling. It has 10 aspects. Those are: (1) psychological development, (2) violence, (3) divorce, (4), busy working 
parents, (5), addicted to alcoholic beverages (6), psychotropic drugs, (6) moral damage, (7) bullying in schools, (8) people with HIV / AIDS, (10), lazy to learn. There are 7 aspects that brings to the crisis, including (1) psychological development, (2) violence, (3) divorce, (4) parent's busyness, (5) moral and religious damage, (6) bullying in school, and (7) an increasing threat of HIV / AIDS.

The ten aspects are described in 80 items statement. The analysis test comes to 20 items which are valid and reliable. Is the child experiencing a serious situation in his daily life? Does the child experience severe development? Do you often feel sad every day? Do you often panic? Do you often feel angry? are you traumatized? The item is valid after going through the item analysis test.

Indicators of violence include verbal and non-verbal violence. Verbal violence includes questions: do you experience verbal violence? Do you experience physical violence? Does violence increase every day? do you experience violence on social media? Do you experience violence at home? After carrying out the analysis test, the valid item is, are you experiencing verbal violence? Are you experiencing physical violence?

Indicator of the busyness of parents working and ignoring children with descriptors of neglect for the children. The items developed include, are parents busy working outside the home? Are children experiencing neglected? Do children feel neglected by parents? Do children tell the story of their lives to parents? Are parents to spend time with children? Do children feel safe playing around their home environment? Are parents often worried about the safety of children outside the home? Are parents' concerns increasingly high for children's safety? Valid items are as to children experiencing omission by parents? Do parents spend time with children? Do parents worry about your security outside the home? Are parents' concerns about the safety of children getting higher? Are you thanking parents?

The divorce indicator covers aspects of family breakdown with question items such as, does your family have a breakdown? Does your family have harmonious communication? is your family having no communication? Are you from a broken home family? Is family divorce a common thing? Is family divorce something disgraceful? Is family divorce something you hated? The valid results, is your family experiencing a rift? Is family divorce normal?

Indicators of moral damage include whether moral damage continues to increase every day? do children often go against parents? do children often hurt their friends? do children imitate value-free live style? do the children act as they please? The valid result is that is moral damage increasing every day?

Bullying indicators in schools in which the items developed are, is the climate in the school full of threats? Is the school situation pleasant? Does the teacher teach patiently? Is the teacher often angry and threatening? Do you often fight with friends? Do you have good friends in school? are you happy to attend school? Are you afraid to come to school? Is school violence increasing every day? Valid items after the validity test Are you hurting friends? Is the climate in the school full of threats?

Indicators of HIV are that are HIV / AIDS sufferers increasing in the environment around you? Is the number of sufferers of HIV / AIDS more and more in your city? Is free sex behavior increasing in your area? Are people indifferent to free sex? Valid items are that are increasing numbers of people with HIV / AIDS in your city? Does free sex increase in your area?

Indicator of alcoholic addiction is that have you ever drunk or contain alcoholic beverages? do you drink alcoholic drinks to eliminate the problem?

Indicators for laziness. Do children often play online games rather than reading books? Do children often spend time watching television? Do children use free time playing gadgets? Do they have dreams for future? Have children aspirations for the future? Are children desperate and have no ideals? 
Measuring instruments developed through favorable and unfavorable items. The goal is to obtain the crisis need assessment which has been tested for validity and reliability. Interpretation results use SPSS 15. The following are the results of validity and reliability tests conducted by the researcher:

Test Empirical validity by using Product Moment correlation

The empirical validity test conducted by the researcher used the product moment correlation test with the help of SPSS.15. product moment correlation in the validity test is a measurement of item validity. This test will correlate between item scores and total scores on each item. The validity coefficient must be interpreted from the coefficient of determination, which is a number that shows the proportion (percentage) of the variance of a variable that can be explained from other variables (Solichin, 2017). The items tested for validity are 80 and there are 20 valid and reliable items. Data is said to be valid if the value of $R$ count $>R$ table. Determination of the number of $R$ tables is determined based on the determined number of samples of 75 students. So that it can be seen that the $R$ table value for DF N-2 is 0.2272 . If $R$ count $>0.2272$ then the item is said to be valid. Conversely, if the $\mathrm{R}$ count $<0.2272$ then the item item is invalid.

Table 1 Hasil analisis product moment

\begin{tabular}{|c|c|c|c|c|c|c|c|c|}
\hline Item & $\begin{array}{c}\text { Explanati } \\
\text { on }\end{array}$ & $\begin{array}{c}\mathbf{R} \\
\text { count }\end{array}$ & Item & $\begin{array}{l}\text { Explanati } \\
\text { on }\end{array}$ & $\begin{array}{c}\mathrm{R} \\
\text { count }\end{array}$ & Item & $\begin{array}{l}\text { Explanati } \\
\text { on }\end{array}$ & $\begin{array}{c}\mathrm{R} \\
\text { count }\end{array}$ \\
\hline \multirow[t]{3}{*}{ item_1 } & $\begin{array}{c}\text { Pearson } \\
\text { Correlation }\end{array}$ & 1 & $\begin{array}{l}\text { item }_{-} \\
28\end{array}$ & $\begin{array}{c}\text { Pearson } \\
\text { Correlation }\end{array}$ & $\begin{array}{c}0,05 \\
4\end{array}$ & $\begin{array}{c}\text { item } \\
55\end{array}$ & $\begin{array}{c}\text { Pearson } \\
\text { Correlation }\end{array}$ & $\begin{array}{l}, 519 \text { (* }^{*} \\
*)\end{array}$ \\
\hline & $\begin{array}{l}\text { Sig. (2- } \\
\text { tailed) }\end{array}$ & & & $\begin{array}{l}\text { Sig. (2- } \\
\text { tailed) }\end{array}$ & $\begin{array}{l}0,64 \\
6\end{array}$ & & $\begin{array}{l}\text { Sig. (2- } \\
\text { tailed) }\end{array}$ & 0 \\
\hline & $\mathrm{N}$ & 75 & & $\mathrm{~N}$ & 75 & & $\mathrm{~N}$ & 75 \\
\hline \multirow[t]{3}{*}{$\begin{array}{c}\text { item } \\
2\end{array}$} & $\begin{array}{c}\text { Pearson } \\
\text { Correlation }\end{array}$ & $\begin{array}{l}, 484\left(^{*}\right. \\
*)\end{array}$ & $\begin{array}{l}\text { item } \\
29\end{array}$ & $\begin{array}{c}\text { Pearson } \\
\text { Correlation }\end{array}$ & $\begin{array}{l}0,10 \\
3\end{array}$ & $\begin{array}{c}\text { item_ } \\
56\end{array}$ & $\begin{array}{c}\text { Pearson } \\
\text { Correlation }\end{array}$ & 0,039 \\
\hline & $\begin{array}{l}\text { Sig. (2- } \\
\text { tailed) }\end{array}$ & 0 & & $\begin{array}{l}\text { Sig. (2- } \\
\text { tailed) }\end{array}$ & 0,38 & & $\begin{array}{l}\text { Sig. (2- } \\
\text { tailed) }\end{array}$ & 0,742 \\
\hline & $\mathrm{N}$ & 75 & & $\mathrm{~N}$ & 75 & & $\mathrm{~N}$ & 75 \\
\hline \multirow[t]{3}{*}{$\begin{array}{c}\text { item } \\
3\end{array}$} & $\begin{array}{c}\text { Pearson } \\
\text { Correlation }\end{array}$ & $\begin{array}{l}\text {,295(* } \\
\text { ) }\end{array}$ & $\begin{array}{l}\text { item }_{-} \\
30\end{array}$ & $\begin{array}{c}\text { Pearson } \\
\text { Correlation }\end{array}$ & $\begin{array}{l}0,20 \\
1\end{array}$ & $\begin{array}{l}\text { item_ }_{5} \\
57\end{array}$ & $\begin{array}{c}\text { Pearson } \\
\text { Correlation }\end{array}$ & $-0,14$ \\
\hline & $\begin{array}{l}\text { Sig. (2- } \\
\text { tailed) }\end{array}$ & 0,01 & & $\begin{array}{l}\text { Sig. (2- } \\
\text { tailed) }\end{array}$ & $\begin{array}{l}0,08 \\
4\end{array}$ & & $\begin{array}{l}\text { Sig. (2- } \\
\text { tailed) }\end{array}$ & 0,231 \\
\hline & $\mathrm{N}$ & 75 & & $\mathrm{~N}$ & 75 & & $\mathrm{~N}$ & 75 \\
\hline \multirow[t]{3}{*}{$\begin{array}{c}\text { item_- } \\
4\end{array}$} & $\begin{array}{c}\text { Pearson } \\
\text { Correlation }\end{array}$ & $\begin{array}{l}, 443\left(^{*}\right. \\
*)\end{array}$ & $\begin{array}{l}\text { item }_{-} \\
31\end{array}$ & $\begin{array}{c}\text { Pearson } \\
\text { Correlation }\end{array}$ & $\begin{array}{l}, 242( \\
*)\end{array}$ & $\begin{array}{l}\text { item }_{-} \\
58\end{array}$ & $\begin{array}{c}\text { Pearson } \\
\text { Correlation }\end{array}$ & $\begin{array}{c}- \\
0,141\end{array}$ \\
\hline & $\begin{array}{l}\text { Sig. (2- } \\
\text { tailed) }\end{array}$ & 0 & & $\begin{array}{l}\text { Sig. (2- } \\
\text { tailed) }\end{array}$ & $\begin{array}{l}0,03 \\
6\end{array}$ & & $\begin{array}{l}\text { Sig. (2- } \\
\text { tailed) }\end{array}$ & 0,228 \\
\hline & $\mathrm{N}$ & 75 & & $\mathrm{~N}$ & 75 & & $\mathrm{~N}$ & 75 \\
\hline \multirow[t]{3}{*}{$\begin{array}{c}\text { item } \\
5\end{array}$} & $\begin{array}{c}\text { Pearson } \\
\text { Correlation }\end{array}$ & $\begin{array}{c}- \\
0,008\end{array}$ & $\begin{array}{l}\text { item } \\
32\end{array}$ & $\begin{array}{c}\text { Pearson } \\
\text { Correlation }\end{array}$ & $\begin{array}{c}- \\
0,112\end{array}$ & $\begin{array}{c}\text { item }_{-} \\
59\end{array}$ & $\begin{array}{c}\text { Pearson } \\
\text { Correlation }\end{array}$ & $\begin{array}{l}, 543\left(^{*}\right. \\
*\end{array}$ \\
\hline & $\begin{array}{l}\text { Sig. (2- } \\
\text { tailed) }\end{array}$ & 0,947 & & $\begin{array}{l}\text { Sig. (2- } \\
\text { tailed) }\end{array}$ & $\begin{array}{l}0,33 \\
8\end{array}$ & & $\begin{array}{l}\text { Sig. (2- } \\
\text { tailed) }\end{array}$ & 0 \\
\hline & $\mathrm{N}$ & 75 & & $\mathrm{~N}$ & 75 & & $\mathrm{~N}$ & 75 \\
\hline \multirow[t]{3}{*}{$\begin{array}{c}\text { item }_{-} \\
6\end{array}$} & $\begin{array}{c}\text { Pearson } \\
\text { Correlation }\end{array}$ & 0,078 & $\begin{array}{l}\text { item }_{-} \\
33\end{array}$ & $\begin{array}{c}\text { Pearson } \\
\text { Correlation }\end{array}$ & $\begin{array}{c}- \\
0,135\end{array}$ & $\begin{array}{c}\text { item } \\
60\end{array}$ & $\begin{array}{c}\text { Pearson } \\
\text { Correlation }\end{array}$ & 0,168 \\
\hline & $\begin{array}{l}\text { Sig. (2- } \\
\text { tailed) }\end{array}$ & 0,507 & & $\begin{array}{l}\text { Sig. (2- } \\
\text { tailed) }\end{array}$ & 0,25 & & $\begin{array}{l}\text { Sig. (2- } \\
\text { tailed) }\end{array}$ & 0,15 \\
\hline & $\mathrm{N}$ & 75 & & $\mathrm{~N}$ & 75 & & $\mathrm{~N}$ & 75 \\
\hline \multirow[t]{2}{*}{$\begin{array}{c}\text { item } \\
7\end{array}$} & $\begin{array}{c}\text { Pearson } \\
\text { Correlation }\end{array}$ & (a) & $\begin{array}{l}\text { item_ } \\
34\end{array}$ & $\begin{array}{c}\text { Pearson } \\
\text { Correlation }\end{array}$ & $\begin{array}{l}, 242( \\
*)\end{array}$ & $\begin{array}{c}\text { item_ } \\
61\end{array}$ & $\begin{array}{c}\text { Pearson } \\
\text { Correlation }\end{array}$ & $\begin{array}{c}- \\
0,041\end{array}$ \\
\hline & Sig. (2- & . & & Sig. (2- & 0,03 & & Sig. (2- & 0,727 \\
\hline
\end{tabular}


RedWhitepress Global Conferences Series: Social Sciences, Education and Humanities (GCSSSEH), Volume 3, 2019

\begin{tabular}{|c|c|c|c|c|c|c|c|c|}
\hline & tailed) & & & tailed) & 6 & & tailed) & \\
\hline & $\mathrm{N}$ & 75 & & $\mathrm{~N}$ & 75 & & $\mathrm{~N}$ & 75 \\
\hline \multirow[t]{3}{*}{$\begin{array}{l}\text { item }_{-} \\
8\end{array}$} & $\begin{array}{c}\text { Pearson } \\
\text { Correlation }\end{array}$ & 0,214 & $\begin{array}{l}\text { item }_{-} \\
35\end{array}$ & $\begin{array}{c}\text { Pearson } \\
\text { Correlation }\end{array}$ & 0,22 & $\begin{array}{l}\text { item } \\
62\end{array}$ & $\begin{array}{c}\text { Pearson } \\
\text { Correlation }\end{array}$ & $\begin{array}{c}- \\
0,115\end{array}$ \\
\hline & $\begin{array}{l}\text { Sig. (2- } \\
\text { tailed) }\end{array}$ & 0,065 & & $\begin{array}{l}\text { Sig. (2- } \\
\text { tailed) }\end{array}$ & $\begin{array}{l}0,05 \\
8\end{array}$ & & $\begin{array}{l}\text { Sig. (2- } \\
\text { tailed) }\end{array}$ & 0,325 \\
\hline & $\mathrm{N}$ & 75 & & $\mathrm{~N}$ & 75 & & $\mathrm{~N}$ & 75 \\
\hline \multirow[t]{3}{*}{$\begin{array}{c}\text { item } \\
9\end{array}$} & $\begin{array}{c}\text { Pearson } \\
\text { Correlation }\end{array}$ & $\begin{array}{c}- \\
0,037\end{array}$ & $\begin{array}{l}\text { item }_{3} \\
36\end{array}$ & $\begin{array}{c}\text { Pearson } \\
\text { Correlation }\end{array}$ & $\begin{array}{l}, 291( \\
*)\end{array}$ & $\begin{array}{l}\text { item_ } \\
63\end{array}$ & $\begin{array}{c}\text { Pearson } \\
\text { Correlation }\end{array}$ & 0,119 \\
\hline & $\begin{array}{l}\text { Sig. (2- } \\
\text { tailed) }\end{array}$ & 0,754 & & $\begin{array}{l}\text { Sig. (2- } \\
\text { tailed) }\end{array}$ & $\begin{array}{l}0,01 \\
1\end{array}$ & & $\begin{array}{l}\text { Sig. (2- } \\
\text { tailed) }\end{array}$ & 0,309 \\
\hline & $\mathrm{N}$ & 75 & & $\mathrm{~N}$ & 75 & & $\mathrm{~N}$ & 75 \\
\hline \multirow[t]{3}{*}{$\begin{array}{l}\text { item } \\
10\end{array}$} & $\begin{array}{c}\text { Pearson } \\
\text { Correlation }\end{array}$ & $\begin{array}{c}- \\
0,067\end{array}$ & $\begin{array}{l}\text { item }_{-} \\
37\end{array}$ & $\begin{array}{c}\text { Pearson } \\
\text { Correlation }\end{array}$ & 0,04 & $\begin{array}{l}\text { item_ } \\
64\end{array}$ & $\begin{array}{c}\text { Pearson } \\
\text { Correlation }\end{array}$ & ,242(* \\
\hline & $\begin{array}{l}\text { Sig. (2- } \\
\text { tailed) }\end{array}$ & 0,568 & & $\begin{array}{l}\text { Sig. (2- } \\
\text { tailed) }\end{array}$ & $\begin{array}{l}0,73 \\
5\end{array}$ & & $\begin{array}{l}\text { Sig. (2- } \\
\text { tailed) }\end{array}$ & 0,036 \\
\hline & $\mathrm{N}$ & 75 & & $\mathrm{~N}$ & 75 & & $\mathrm{~N}$ & 75 \\
\hline \multirow[t]{3}{*}{$\begin{array}{c}\text { item } \\
11\end{array}$} & $\begin{array}{c}\text { Pearson } \\
\text { Correlation }\end{array}$ & $\begin{array}{c}- \\
0,048\end{array}$ & $\begin{array}{l}\text { item }_{3} \\
38\end{array}$ & $\begin{array}{c}\text { Pearson } \\
\text { Correlation }\end{array}$ & $\begin{array}{l}, 275( \\
*)\end{array}$ & $\begin{array}{c}\text { item_ } \\
65\end{array}$ & $\begin{array}{c}\text { Pearson } \\
\text { Correlation }\end{array}$ & $\begin{array}{l}, 425\left(^{*}\right. \\
\left.{ }^{*}\right)\end{array}$ \\
\hline & $\begin{array}{l}\text { Sig. (2- } \\
\text { tailed) }\end{array}$ & 0,682 & & $\begin{array}{l}\text { Sig. (2- } \\
\text { tailed) }\end{array}$ & $\begin{array}{l}0,01 \\
7\end{array}$ & & $\begin{array}{l}\text { Sig. (2- } \\
\text { tailed) }\end{array}$ & 0 \\
\hline & $\mathrm{N}$ & 75 & & N & 75 & & N & 75 \\
\hline \multirow[t]{3}{*}{$\begin{array}{c}\text { item } \\
12\end{array}$} & $\begin{array}{c}\text { Pearson } \\
\text { Correlation }\end{array}$ & $\begin{array}{c}- \\
0,012\end{array}$ & $\begin{array}{l}\text { item } \\
39\end{array}$ & $\begin{array}{c}\text { Pearson } \\
\text { Correlation }\end{array}$ & $\begin{array}{c}0,13 \\
4\end{array}$ & $\begin{array}{c}\text { item_ } \\
66\end{array}$ & $\begin{array}{c}\text { Pearson } \\
\text { Correlation }\end{array}$ & 0,153 \\
\hline & $\begin{array}{l}\text { Sig. (2- } \\
\text { tailed) }\end{array}$ & 0,918 & & $\begin{array}{l}\text { Sig. (2- } \\
\text { tailed) }\end{array}$ & $\begin{array}{l}0,25 \\
3\end{array}$ & & $\begin{array}{l}\text { Sig. (2- } \\
\text { tailed) }\end{array}$ & 0,189 \\
\hline & $\mathrm{N}$ & 75 & & $\mathrm{~N}$ & 75 & & $\mathrm{~N}$ & 75 \\
\hline \multirow[t]{3}{*}{$\begin{array}{l}\text { item } \\
13\end{array}$} & $\begin{array}{c}\text { Pearson } \\
\text { Correlation }\end{array}$ & 0,036 & $\begin{array}{l}\text { item_ } \\
40\end{array}$ & $\begin{array}{c}\text { Pearson } \\
\text { Correlation }\end{array}$ & $\begin{array}{c}- \\
0,088\end{array}$ & $\begin{array}{l}\text { item_- }_{-} \\
67\end{array}$ & $\begin{array}{c}\text { Pearson } \\
\text { Correlation }\end{array}$ & 0,105 \\
\hline & $\begin{array}{l}\text { Sig. (2- } \\
\text { tailed) }\end{array}$ & 0,757 & & $\begin{array}{l}\text { Sig. (2- } \\
\text { tailed) }\end{array}$ & $\begin{array}{c}0,45 \\
4\end{array}$ & & $\begin{array}{l}\text { Sig. (2- } \\
\text { tailed) }\end{array}$ & 0,368 \\
\hline & $\mathrm{N}$ & 75 & & $\mathrm{~N}$ & 75 & & $\mathrm{~N}$ & 75 \\
\hline \multirow[t]{3}{*}{$\begin{array}{c}\text { item }_{-} \\
14\end{array}$} & $\begin{array}{c}\text { Pearson } \\
\text { Correlation }\end{array}$ & 0,133 & $\begin{array}{l}\text { item_ } \\
41\end{array}$ & $\begin{array}{c}\text { Pearson } \\
\text { Correlation }\end{array}$ & $\begin{array}{l}0,14 \\
1\end{array}$ & $\begin{array}{c}\text { item_ } \\
68\end{array}$ & $\begin{array}{c}\text { Pearson } \\
\text { Correlation }\end{array}$ & $\begin{array}{c}- \\
0,191\end{array}$ \\
\hline & $\begin{array}{l}\text { Sig. (2- } \\
\text { tailed) }\end{array}$ & 0,255 & & $\begin{array}{l}\text { Sig. (2- } \\
\text { tailed) }\end{array}$ & $\begin{array}{l}0,22 \\
8\end{array}$ & & $\begin{array}{l}\text { Sig. (2- } \\
\text { tailed) }\end{array}$ & 0,1 \\
\hline & $\mathrm{N}$ & 75 & & $\mathrm{~N}$ & 75 & & $\mathrm{~N}$ & 75 \\
\hline \multirow[t]{3}{*}{$\begin{array}{l}\text { item } \\
15\end{array}$} & $\begin{array}{c}\text { Pearson } \\
\text { Correlation }\end{array}$ & $\begin{array}{c}- \\
0,053\end{array}$ & $\begin{array}{c}\text { item }_{-} \\
42\end{array}$ & $\begin{array}{c}\text { Pearson } \\
\text { Correlation }\end{array}$ & $\begin{array}{l}0,12 \\
5\end{array}$ & $\begin{array}{c}\text { item } \\
69\end{array}$ & $\begin{array}{c}\text { Pearson } \\
\text { Correlation }\end{array}$ & $\begin{array}{c}- \\
0,055\end{array}$ \\
\hline & $\begin{array}{l}\text { Sig. (2- } \\
\text { tailed) }\end{array}$ & 0,654 & & $\begin{array}{l}\text { Sig. (2- } \\
\text { tailed) }\end{array}$ & $\begin{array}{l}0,28 \\
6\end{array}$ & & $\begin{array}{l}\text { Sig. (2- } \\
\text { tailed) }\end{array}$ & 0,642 \\
\hline & $\mathrm{N}$ & 75 & & $\mathrm{~N}$ & 75 & & $\mathrm{~N}$ & 75 \\
\hline \multirow[t]{3}{*}{$\begin{array}{l}\text { item } \\
16\end{array}$} & $\begin{array}{c}\text { Pearson } \\
\text { Correlation }\end{array}$ & $\begin{array}{c}- \\
0,044\end{array}$ & $\begin{array}{c}\text { item_ } \\
43\end{array}$ & $\begin{array}{c}\text { Pearson } \\
\text { Correlation }\end{array}$ & $\begin{array}{c}- \\
0,058\end{array}$ & $\begin{array}{l}\text { item_ } \\
70\end{array}$ & $\begin{array}{c}\text { Pearson } \\
\text { Correlation }\end{array}$ & $\begin{array}{c}- \\
0,223\end{array}$ \\
\hline & $\begin{array}{l}\text { Sig. (2- } \\
\text { tailed) }\end{array}$ & 0,706 & & $\begin{array}{l}\text { Sig. (2- } \\
\text { tailed) }\end{array}$ & $\begin{array}{c}0,62 \\
2\end{array}$ & & $\begin{array}{l}\text { Sig. (2- } \\
\text { tailed) }\end{array}$ & 0,054 \\
\hline & $\mathrm{N}$ & 75 & & $\mathrm{~N}$ & 75 & & $\mathrm{~N}$ & 75 \\
\hline $\begin{array}{l}\text { item } \\
17\end{array}$ & $\begin{array}{c}\text { Pearson } \\
\text { Correlation }\end{array}$ & .(a) & $\begin{array}{c}\text { item_ } \\
44\end{array}$ & $\begin{array}{c}\text { Pearson } \\
\text { Correlation }\end{array}$ & $\begin{array}{l}0,03 \\
7\end{array}$ & $\begin{array}{c}\text { item }_{-} \\
71\end{array}$ & $\begin{array}{c}\text { Pearson } \\
\text { Correlation }\end{array}$ & 0,025 \\
\hline & $\begin{array}{l}\text { Sig. (2- } \\
\text { tailed) }\end{array}$ & & & $\begin{array}{l}\text { Sig. (2- } \\
\text { tailed) }\end{array}$ & $\begin{array}{l}0,75 \\
3\end{array}$ & & $\begin{array}{l}\text { Sig. (2- } \\
\text { tailed) }\end{array}$ & 0,831 \\
\hline
\end{tabular}


RedWhitepress Global Conferences Series: Social Sciences, Education and Humanities (GCSSSEH), Volume 3, 2019

\begin{tabular}{|c|c|c|c|c|c|c|c|c|}
\hline & $\mathrm{N}$ & 75 & & $\mathrm{~N}$ & 75 & & $\mathrm{~N}$ & 75 \\
\hline \multirow[t]{3}{*}{$\begin{array}{c}\text { item }_{-} \\
18\end{array}$} & $\begin{array}{c}\text { Pearson } \\
\text { Correlation }\end{array}$ & (a) & $\begin{array}{c}\text { item } \\
45\end{array}$ & $\begin{array}{c}\text { Pearson } \\
\text { Correlation }\end{array}$ & $\begin{array}{l}0,12 \\
2\end{array}$ & $\begin{array}{c}\text { item_ } \\
72\end{array}$ & $\begin{array}{c}\text { Pearson } \\
\text { Correlation }\end{array}$ & 0,082 \\
\hline & $\begin{array}{l}\text { Sig. (2- } \\
\text { tailed) }\end{array}$ & & & $\begin{array}{l}\text { Sig. (2- } \\
\text { tailed) }\end{array}$ & $\begin{array}{l}0,29 \\
9\end{array}$ & & $\begin{array}{l}\text { Sig. (2- } \\
\text { tailed) }\end{array}$ & 0,487 \\
\hline & $\mathrm{N}$ & 75 & & $\mathrm{~N}$ & 75 & & $\mathrm{~N}$ & 75 \\
\hline \multirow[t]{3}{*}{$\begin{array}{c}\text { item } \\
19\end{array}$} & $\begin{array}{c}\text { Pearson } \\
\text { Correlation }\end{array}$ & (a) & $\begin{array}{l}\text { item } \\
46\end{array}$ & $\begin{array}{c}\text { Pearson } \\
\text { Correlation }\end{array}$ & $\begin{array}{c}- \\
0,177\end{array}$ & item_ & $\begin{array}{c}\text { Pearson } \\
\text { Correlation }\end{array}$ & $\begin{array}{c}, 295\left(^{*}\right. \\
)\end{array}$ \\
\hline & $\begin{array}{l}\text { Sig. (2- } \\
\text { tailed) }\end{array}$ & & & $\begin{array}{l}\text { Sig. (2- } \\
\text { tailed) }\end{array}$ & 0,13 & & $\begin{array}{l}\text { Sig. (2- } \\
\text { tailed) }\end{array}$ & 0,01 \\
\hline & $\mathrm{N}$ & 75 & & $\mathrm{~N}$ & 75 & & $\mathrm{~N}$ & 75 \\
\hline \multirow[t]{3}{*}{$\begin{array}{c}\text { item } \\
20\end{array}$} & $\begin{array}{c}\text { Pearson } \\
\text { Correlation }\end{array}$ & 0,086 & $\begin{array}{l}\text { item }_{-} \\
47\end{array}$ & $\begin{array}{c}\text { Pearson } \\
\text { Correlation }\end{array}$ & $\begin{array}{l}0,03 \\
7\end{array}$ & $\begin{array}{l}\text { item_ } \\
74\end{array}$ & $\begin{array}{c}\text { Pearson } \\
\text { Correlation }\end{array}$ & 0,104 \\
\hline & $\begin{array}{l}\text { Sig. (2- } \\
\text { tailed) }\end{array}$ & 0,461 & & $\begin{array}{l}\text { Sig. (2- } \\
\text { tailed) }\end{array}$ & $\begin{array}{l}0,75 \\
5\end{array}$ & & $\begin{array}{l}\text { Sig. (2- } \\
\text { tailed) }\end{array}$ & 0,373 \\
\hline & $\mathrm{N}$ & 75 & & $\mathrm{~N}$ & 75 & & $\mathrm{~N}$ & 75 \\
\hline \multirow[t]{3}{*}{$\begin{array}{c}\text { item } \\
21\end{array}$} & $\begin{array}{c}\text { Pearson } \\
\text { Correlation }\end{array}$ & $\begin{array}{c}, 248\left(^{*}\right. \\
)\end{array}$ & $\begin{array}{l}\text { item }_{-} \\
48\end{array}$ & $\begin{array}{c}\text { Pearson } \\
\text { Correlation }\end{array}$ & $\begin{array}{c}- \\
0,182\end{array}$ & $\begin{array}{l}\text { item }_{-} \\
75\end{array}$ & $\begin{array}{c}\text { Pearson } \\
\text { Correlation }\end{array}$ & $\frac{-}{400(* *)}$ \\
\hline & $\begin{array}{l}\text { Sig. (2- } \\
\text { tailed) }\end{array}$ & 0,032 & & $\begin{array}{l}\text { Sig. (2- } \\
\text { tailed) }\end{array}$ & $\begin{array}{l}0,11 \\
9\end{array}$ & & $\begin{array}{l}\text { Sig. (2- } \\
\text { tailed) }\end{array}$ & 0 \\
\hline & $\mathrm{N}$ & 75 & & $\mathrm{~N}$ & 75 & & $\mathrm{~N}$ & 75 \\
\hline \multirow[t]{3}{*}{$\begin{array}{c}\text { item } \\
22\end{array}$} & $\begin{array}{c}\text { Pearson } \\
\text { Correlation }\end{array}$ & $\begin{array}{l}, 2566^{*} \\
)\end{array}$ & $\begin{array}{l}\text { item_ } \\
49\end{array}$ & $\begin{array}{c}\text { Pearson } \\
\text { Correlation }\end{array}$ & $\begin{array}{c}- \\
0,008\end{array}$ & $\begin{array}{c}\text { item }_{-} \\
76\end{array}$ & $\begin{array}{c}\text { Pearson } \\
\text { Correlation }\end{array}$ & $\begin{array}{c}- \\
\left., 2544^{*}\right)\end{array}$ \\
\hline & $\begin{array}{l}\text { Sig. (2- } \\
\text { tailed) }\end{array}$ & 0,027 & & $\begin{array}{l}\text { Sig. (2- } \\
\text { tailed) }\end{array}$ & $\begin{array}{c}0,94 \\
6\end{array}$ & & $\begin{array}{l}\text { Sig. (2- } \\
\text { tailed) }\end{array}$ & 0,028 \\
\hline & N & 75 & & $\mathrm{~N}$ & 75 & & $\mathrm{~N}$ & 75 \\
\hline \multirow[t]{3}{*}{$\begin{array}{c}\text { item } \\
23\end{array}$} & $\begin{array}{c}\text { Pearson } \\
\text { Correlation }\end{array}$ & $\begin{array}{l}, 324\left(^{*}\right. \\
*)\end{array}$ & $\begin{array}{c}\text { item } \\
50\end{array}$ & $\begin{array}{c}\text { Pearson } \\
\text { Correlation }\end{array}$ & $\begin{array}{l}0,09 \\
8\end{array}$ & $\begin{array}{c}\text { item_ } \\
77\end{array}$ & $\begin{array}{c}\text { Pearson } \\
\text { Correlation }\end{array}$ & 0,169 \\
\hline & $\begin{array}{l}\text { Sig. (2- } \\
\text { tailed) }\end{array}$ & 0,005 & & $\begin{array}{l}\text { Sig. (2- } \\
\text { tailed) }\end{array}$ & $\begin{array}{l}0,40 \\
4\end{array}$ & & $\begin{array}{l}\text { Sig. (2- } \\
\text { tailed) }\end{array}$ & 0,148 \\
\hline & N & 75 & & $\mathrm{~N}$ & 75 & & $\mathrm{~N}$ & 75 \\
\hline \multirow[t]{3}{*}{$\begin{array}{c}\text { item }_{-} \\
24\end{array}$} & $\begin{array}{c}\text { Pearson } \\
\text { Correlation }\end{array}$ & 0,138 & $\begin{array}{l}\text { item }_{-} \\
51\end{array}$ & $\begin{array}{c}\text { Pearson } \\
\text { Correlation }\end{array}$ & $\begin{array}{l}0,18 \\
7\end{array}$ & $\begin{array}{c}\text { item_ } \\
78\end{array}$ & $\begin{array}{c}\text { Pearson } \\
\text { Correlation }\end{array}$ & $\begin{array}{l}, 419\left(^{*}\right. \\
*)\end{array}$ \\
\hline & $\begin{array}{l}\text { Sig. (2- } \\
\text { tailed) }\end{array}$ & 0,237 & & $\begin{array}{l}\text { Sig. (2- } \\
\text { tailed) }\end{array}$ & $\begin{array}{l}0,10 \\
8\end{array}$ & & $\begin{array}{l}\text { Sig. (2- } \\
\text { tailed) }\end{array}$ & 0 \\
\hline & $\mathrm{N}$ & 75 & & $\mathrm{~N}$ & 75 & & $\mathrm{~N}$ & 75 \\
\hline \multirow[t]{3}{*}{$\begin{array}{c}\text { item } \\
25\end{array}$} & $\begin{array}{c}\text { Pearson } \\
\text { Correlation }\end{array}$ & $\begin{array}{l}, 345\left(^{*}\right. \\
*)\end{array}$ & $\begin{array}{c}\text { item }_{-} \\
52\end{array}$ & $\begin{array}{c}\text { Pearson } \\
\text { Correlation }\end{array}$ & $\begin{array}{l}0,06 \\
3\end{array}$ & $\begin{array}{l}\text { item_ } \\
79\end{array}$ & $\begin{array}{c}\text { Pearson } \\
\text { Correlation }\end{array}$ & $\begin{array}{c}- \\
0,025\end{array}$ \\
\hline & $\begin{array}{l}\text { Sig. (2- } \\
\text { tailed) }\end{array}$ & 0,002 & & $\begin{array}{l}\text { Sig. (2- } \\
\text { tailed) }\end{array}$ & $\begin{array}{c}0,59 \\
1\end{array}$ & & $\begin{array}{l}\text { Sig. (2- } \\
\text { tailed) }\end{array}$ & 0,829 \\
\hline & N & 75 & & N & 75 & & N & 75 \\
\hline \multirow[t]{3}{*}{$\begin{array}{c}\text { item }_{-} \\
26\end{array}$} & $\begin{array}{c}\text { Pearson } \\
\text { Correlation }\end{array}$ & $\begin{array}{c}- \\
0,158\end{array}$ & $\begin{array}{c}\text { item } \\
53\end{array}$ & $\begin{array}{c}\text { Pearson } \\
\text { Correlation }\end{array}$ & $\begin{array}{c}- \\
0,047\end{array}$ & $\begin{array}{c}\text { item }_{-} \\
80\end{array}$ & $\begin{array}{c}\text { Pearson } \\
\text { Correlation }\end{array}$ & $\begin{array}{c}- \\
0,085\end{array}$ \\
\hline & $\begin{array}{l}\text { Sig. (2- } \\
\text { tailed) }\end{array}$ & 0,175 & & $\begin{array}{l}\text { Sig. (2- } \\
\text { tailed) }\end{array}$ & $\begin{array}{c}0,68 \\
8\end{array}$ & & $\begin{array}{l}\text { Sig. (2- } \\
\text { tailed) }\end{array}$ & 0,47 \\
\hline & $\mathrm{N}$ & 75 & & $\mathrm{~N}$ & 75 & & $\mathrm{~N}$ & 75 \\
\hline \multirow[t]{3}{*}{$\begin{array}{c}\text { item } \\
27\end{array}$} & $\begin{array}{c}\text { Pearson } \\
\text { Correlation }\end{array}$ & $\begin{array}{c}- \\
0,158\end{array}$ & $\begin{array}{c}\text { item_ } \\
54\end{array}$ & $\begin{array}{c}\text { Pearson } \\
\text { Correlation }\end{array}$ & $\begin{array}{l}0,19 \\
3\end{array}$ & & & \\
\hline & $\begin{array}{l}\text { Sig. (2- } \\
\text { tailed) }\end{array}$ & 0,175 & & $\begin{array}{l}\text { Sig. (2- } \\
\text { tailed) }\end{array}$ & $\begin{array}{l}0,09 \\
8\end{array}$ & & & \\
\hline & $\mathrm{N}$ & 75 & & $\mathrm{~N}$ & 75 & & & \\
\hline
\end{tabular}


Tabel 2. Assessment (after validation)

** Correlation is significant at the 0.01 level (2-tailed)

* Correlation is significant at the 0.05 level (2-tailed)

\begin{tabular}{|c|c|c|c|c|}
\hline No & Indicator & Descriptor & Questions & $R$ count \\
\hline 1 & $\begin{array}{l}\text { Psychological } \\
\text { development }\end{array}$ & grave & $\begin{array}{c}\text { Does the child experience a serious } \\
\text { situation in his daily life? } \\
\text { Does the child experience severe } \\
\text { development in the soul? } \\
\text { Do you often feel sad? } \\
\text { Do you panic frequently? } \\
\text { Are you easily angry? }\end{array}$ & \\
\hline 2 & Violence & $\begin{array}{l}\text { Verbal and } \\
\text { nonverbal } \\
\text { violence }\end{array}$ & $\begin{array}{l}\text { Do you experience verbal violence? } \\
\text { Do you experience physical violence? } \\
\text { Have you ever experienced trauma }\end{array}$ & \\
\hline 3 & Divorce & $\begin{array}{l}\text { Family } \\
\text { breakdown }\end{array}$ & $\begin{array}{l}\text { Did your family experience cracks? } \\
\text { Is family divorce an ordinary thing? }\end{array}$ & \\
\hline 4 & Moral damage & Moral damage & $\begin{array}{l}\text { Does moral damage continue to } \\
\text { increase every day? }\end{array}$ & \\
\hline 5 & $\begin{array}{l}\text { Bullying in } \\
\text { school }\end{array}$ & $\begin{array}{l}\text { Bulying } \\
\text { Beating } \\
\text { theft }\end{array}$ & $\begin{array}{l}\text { Do you hurt his friends? } \\
\text { Is the climate in the school full of } \\
\text { threats? }\end{array}$ & \\
\hline 6 & $\begin{array}{l}\text { HIV /AIDS } \\
\text { increases }\end{array}$ & $\begin{array}{l}\text { HIV/AIDS } \\
\text { sufferer }\end{array}$ & $\begin{array}{l}\text { Are there more numbers of people } \\
\text { with HIV / AIDS in your city? } \\
\text { Does free sex increase in your area? }\end{array}$ & \\
\hline 7 & $\begin{array}{l}\text { The busyness of } \\
\text { parents works and } \\
\text { ignores children }\end{array}$ & $\begin{array}{l}\text { Neglecting to } \\
\text { children } \\
\text { Abandonment of } \\
\text { children }\end{array}$ & $\begin{array}{l}\text { Do children experience omission by } \\
\text { parents? } \\
\text { Do parents spend time with } \\
\text { children? } \\
\text { Do parents worry about your } \\
\text { security outside the home? }\end{array}$ & \\
\hline & & & $\begin{array}{c}\text { Are parents' concerns increasingly } \\
\text { high for children's safety? } \\
\text { Do you thank parents }\end{array}$ & \\
\hline
\end{tabular}

Test reliability using the Cronbach's Alpha method

Reliability test is done by using the Cronbach alpha formula. The reliability testing process is carried out by collecting valid items and then performing the reliability test. Following is the test statistics using Cronbach Alpha:

Table 3 Reliability Statistics

\begin{tabular}{cc}
\hline Cronbach's & $\mathrm{N}$ of \\
Alpha & Items \\
730 & 20 \\
\hline
\end{tabular}


The table above illustrates that the cronbach alpha value is 0.730 . The value of 0.70 or more is generally accepted as an instrument that has good reliability (Litwin, 1995). The value shown by this instrument test is 0.73 so it can be concluded that this instrument is reliable.

The implication of this finding is that personal assistance to children needs to be developed for self-empowerment and empowerment. Children are crisis prone to aspects of psychological development that feel emergency, panic, sadness and anger and trauma. Counselors need to help provide crisis counseling with individual and group approaches. The crisis counseling strategy with the ACT integrative model ACT model emphasizes Assessment, connecting to support groups, Traumatic stress reactions (ACT) (Yeager \& Roberts, 2015). Development of positive self-concepts with psychodrama techniques (Nurfaizal, 2016).

The findings of this study strengthen the field of social personalities in service delivery by counselors in schools. Personal counseling and guidance material in schools includes enhancing religion and morals, positive self-concepts, self-esteem, respecting parents and families, assertive behavioral training and ways to avoid the risk of HIV / AIDS. Strengthening children's religion can be through strengthening faith and piety to God, doing good and living in the pleasure of God (Gustini, 2016). The congregational prayer program in schools needs to be supported by counselors, as well as the involvement of parents at home to supervise children in worship. No matter how busy parents are at work, it is important to remind children to do five times of prayer and to recite the Qur'an. and to fortify children from moral damage that continues to increase every day. With children holding fast to the rope of God, they will get a sense of security everywhere.

\section{Conclusions}

The results of developing need assessment Guidance and crisis counseling consist of aspects (1) psychological development, (2). violence, (3) divorce, (4) busy life of parents, (5) moral and religious damage, (6) bullying in schools, (7) increasing threat of HIV / AIDS. There are 20 valid and reliable items. Counselors can use need assessment to find out the crisis conditions encountered by individuals.

Materials that need to be developed by counselors in schools include personal social fields, including moral and religious enhancement, positive self-concepts, self-esteem, respect for parents and families, assertive behavior training and ways to avoid the risk of HIV / AIDS.

The crisis counseling and guidance need assessment needs to be carried out on more respondents to map the counselee needs of the broader counseling and guidance Crisis, so that it can become material in the development of crisis counseling and guidance services in schools.

\section{References}

Bronfenbrenner, U. (1986). Ecology of the family as a context for human development: Research perspectives. Developmental psychology, 22(6), 723.

Etikan, I., Musa, S. A., \& Alkassim, R. S. (2016). Comparison of convenience sampling and purposive sampling. American journal of theoretical and applied statistics, 5(1), 1-4.

Foa, E. B., Chrestman, K. R., \& Gilboa-Schechtman, E. (2008). Prolonged exposure therapy for adolescents with PTSD emotional processing of traumatic experiences, therapist guide: Oxford University Press.

Gustini, N. (2016). Bimbingan dan Konseling melalui Pengembangan Akhlak Mulia Siswa Berbasis Pemikiran Al-Ghazali. Tadris: Jurnal Keguruan dan Ilmu Tarbiyah, 1(1), 1-14.

Kopetz, C., Woerner, J. I., MacPherson, L., Lejuez, C. W., Nelson, C. A., Zeanah, C. H., \& Fox, N. A. (2019). Early psychosocial deprivation and adolescent risk-taking: The role of motivation and executive control. Journal of experimental psychology: general, 148(2), 388.

Litwin, M. S. (1995). How to measure survey reliability and validity (Vol. 7): Sage.

Malawi, I., \& Maruti, E. S. (2009). Evaluasi Pendidikan: CV. AE MEDIA GRAFIKA.

Maté, G., \& Neufeld, G. (2019). Hold on to your kids: Why parents need to matter more than peers: Random House. 
Matondang, Z. (2009). Validitas dan reliabilitas suatu instrumen penelitian. Jurnal Tabularasa, 6(1), 8797.

Novita Luthfiana, D., \& Psi, U. K. S. (2017). Cara Mengatasi Masalah Pada Siswa SMP Yang Terindikasi Conduct Problem. Universitas Muhammadiyah Surakarta.

Nurfaizal, N. (2016). Penggunaan Teknik Psikodrama Untuk Meningkatkan Konsep Diri Siswa. Jurnal Fokus Konseling, 2(2).

Solichin, M. (2017). Analisis Daya Beda Soal, Taraf Kesukaran, Validitas Butir Tes, Interpretasi Hasil Tes dan Validitas Ramalan dalam Evaluasi Pendidikan. Dirāsāt: Jurnal Manajemen dan Pendidikan Islam, 2(2), 192-213.

Yeager, K. R., \& Roberts, A. R. (2015). The ACT Model: Assessment, Crisis Intervention, and Trauma Treatment in the Aftermath of Community Disasters: Crisis Intervention Handbook: Assessment, Treatment and Research.(4th ed.) 